\title{
ESPERIENZE SULLA ELASTICITÀ A TRAZIONE
}

\author{
DEL RAME.
}

No'ta III di GUSTAVO COLONNETTI ${ }^{1}$ )

Le ricerche sperimentali riferite nelle mie due precedenti Note su questo argomento ${ }^{2}$ ) mettono cosi chiaramente in evidenza l'analogia, d'altronde ben nota, che passa tra l'isteresi elastica e l'isteresi magnetica, che il lettore non avrà mancato di istituire spontaneamente un parallelo fra il concetto di deformazione permanente e quello di magnetizzazione residua.

Tale parallelo richiederebbe, per essere svolto a fondo, il tracciamento sperimentale di cicli bilaterali (cioè compresi fra limiti di forza di segni contrarii) che le nostre macchine per le prove dei materiali non permettono assolutamente di eseguire. Nei limiti puramente qualitativi che fin dal principio ho imposto alla mia indagine, il fenomeno può però essere approfondito nel modo seguente.

Uno dei soliti fili di rame, il quale, per un lungo periodo di tempo, aveva servito alle più svariate esperienze, rimanendone energicamente incrudito, venne, il 2 febbraio 1914, assoggettato ad un carico variabile gradualmente (in ragione di circa $50 \mathrm{kgr}$. al minuto primo), alternativamente in senso crescente e decrescente, fra $i$ seguenti limiti:

$$
1200,100,1000,300,800,500,600 \mathrm{kgr} \text {. }
$$

Un tale trattamento può ficeilmente concepirsi come risultante dalla sovrapposizione di un primo carico il quale, raggiunti i $600 \mathrm{kgr}$., si sia mantenuto costante durante tutto il resto dell'esperienża, e di un altro sforzo che diremo, per intenderci,

1) Rendiconti della R. Accademia dei Lincei, vol. XXII, serie 5. a, $10^{\circ}$ sem.

$\left.{ }^{2}\right)$ Nuovo Cimento, serie VI, vol. VII, Gennaio-Febbraio 1914. 
addizionale, il quale varii fra $\mathrm{i}$ seguenti valori:

$$
+600,-500,+400,-300,+200,-100,0 \mathrm{kgr} \text {. }
$$

alternativamente positivi e negativi, e tali che la differenza tra il valore assoluto di uno qualunque di essi ed il valor assoluto del successivo sia una costante positiva.

Il comportamento del materiale si può rilevare così dalla tabella numerica allegata, come dal diagramma disegnato con tratto continuo nella parte sinistra della fig. 1 ; esso ha questo di interessante, che basta a caratterizzare completamente lo

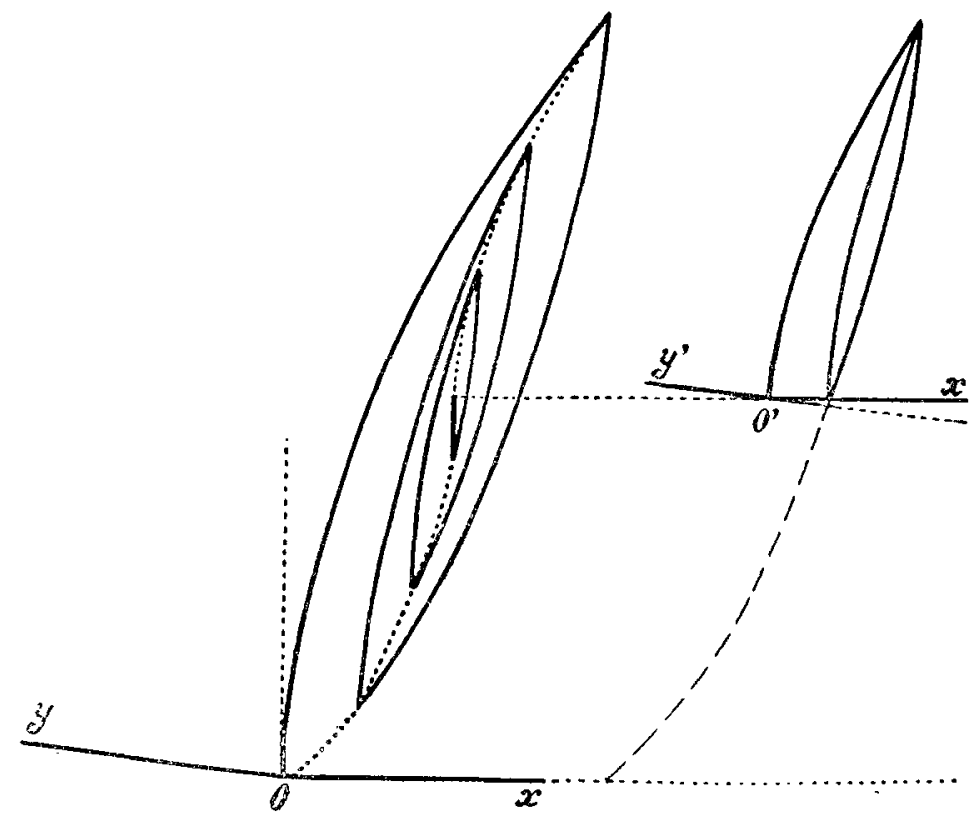

Fig. 1.

stato in cui il materiale si viene a trovare alla fine di questa prima fase (preparatoria) dell' esperienza, nel senso che le leggi generali, che ho gia avuto precedentemente occasione di enunciare (Nota II), bastano da sole a determinare in qual modo il materiale dovrà deformarsi al variare ulteriore del carico applicato. Ed invero, sia che detto carico prenda a crescere, sia che esso prenda a diminuire, debbono man mano chiudersi 
successivamente tanti cicli quanti durante le operazioni precedenti ne sono rimasti di aperti: in altri termini, le due curve di deformazione che si possono descrivere facendo crescere lo sforzo addizionale fino $\mathrm{a}+600 \mathrm{kgr}$, ovvero facendolo decrescere fino a - $600 \mathrm{kgr}$., debbono contenere tutti i successivi punti di regresso corrispondenti rispettivamente ai massimi ovvero ai minimi valori che il carico ha toccati nelle trasformazioni suddescritte, epperò debbono necessariamente presentare l'andamento indicato mediante punteggiate nella figura.

L'esperienza, eseguita nel senso dei carichi crescenti, ha completamente confermato queste previsioni, fornendo valori della deformazione praticamente coincidenti con quelli osservati alla fine delle varie operazioni di carica realizzate durante il periodo preparatorio.

La curva di deformazione che cosi si ottiene non è più pertanto una delle solite curve limiti di un ciclo chiuso: essa presenta, a fronte di quelle, una curvatura assai più sentita, e ricorda, nel suo andamento generale, quella che abbiamo a suo tempo convenuto di chiamare la curva di prima deformazione. Nè è da credersi che si tratti soltanto di una semplice somiglianza di forma: si riscontra qui un vero e proprio rinnovarsi di quei medesimi fenomeni che caratterizzano il comportamento del materiale quando esso vien cimentato per la prima volta. Il cappio ottenuto facendo variare lo sforzo addizionale da $600 \mathrm{kgr}$. a 0 e viceversa (riferito, nella parte destra della figura 1 , ai due nuovi assi $x^{\prime}$ ed $y^{\prime}$, la cui origine $\mathrm{O}^{\prime}$ rappresenta lo stesso speciale stato di deformazione in cui il materiale si trovava alla fine della prima fase dell'esperienza), presenta infatti immutate tutte le caratteristiche dei processi di prima deformazione di cui ci siamo occupati nella Nota I, tanto per ciò che concerne l'esistenza delle deformazioni permanenti, quanto per ciò che riguarda l'intervento di quelle speciali modificazioni, nella deformabilità del materiale, che si sono allora denotate col nome generico di incrudimento.

Senonchè questo incrudimento ci si presenta questa volta completamente giustificato dallo specialissimo trattamento a cui il materiale è stato in precedenza assoggettato. Ed ̀̀ poi 
facile constatare che la stessa deformazione permanente non è qui altro che una semplice manifestazione dei fenomeni di isteresi: se infatti si attribuiscono al carico addizionale valori negativi, la deformazione (intesa sempre contata a partire dallo stato in cui il materiale si trovava alla fine della fase preparatoria) si annulla ben presto ${ }^{4}$ ), poi diviene a sua volta negativa, raggiungendo una grandezza eguale a quella della massima deformazione positiva precisamente quando il carico addizionale diviene eguale a $-600 \mathrm{kgr}^{2}{ }^{2}$ ).

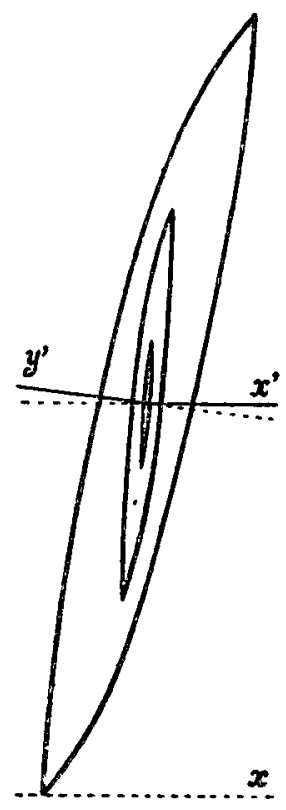

Fig. 2.

Il comportamento del materiale che ha subito la prepara. zione dianzi descritta, per quanto in sostanza non differisca

') L'intensità della forza coercitiva non raggiunge, nel caso concreto, i $10 \mathrm{kgr}$.

$\left.{ }^{2}\right)$ Riprendendo l'esperienza dal principio si verrebbe a realizzare, in un caso anche più generale, quel processo di eliminazione delle deformazioni per alternazioni decrescenti che il Cantone utilizzò sistematicamente nelle sue già citate ricerche. 
da quello che si riscontra nei saggi presi allo stato naturale, cessa pertanto di essere un fatto di eccezione, per rientrare nell'ambito delle leggi generali che regolano i processi di deformazione per cicli chiusi.

Alcune esperienze successive mi hanno permesso di riconoscere che la diversa velocità con cui le operazioni sopra descritte potevano venir realizzate, se influiva sull'entita numerica dei singoli risultati, non alterava però menomamente l'andamento generale dell' esperienza. A questo proposito mi sono sembrati particolarmente degni di essere qui ricordati alcuni cicli (fig. 2) presentanti tutti i caratteri della bilateralità, che jo ho rilevati il 10 febbraio ultimo scorso operando nel solito modo sullo stesso spezzone, dopo di avergli fatte subire tutte le trasformazioni di cui consta la fase preparatoria con la massima velocità che la macchina permette di realizzare quando si rinuncia ad apprezzare le deformazioni, velocità che è di almeno 100 volte più grande di quella adottata nelle esperienze del 2 febbraio.

\begin{tabular}{|c|c|c|c|c|c|c|c|c|c|c|}
\hline \multirow{3}{*}{ Giorno } & \multirow{3}{*}{ Ora } & \multirow{3}{*}{ 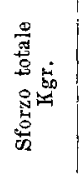 } & \multicolumn{2}{|c|}{ Specchio sinistro } & \multicolumn{2}{|c|}{ Specthio destro } & \multirow{3}{*}{ 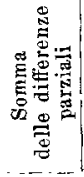 } & \multirow{2}{*}{\multicolumn{2}{|c|}{$\begin{array}{c}\text { Leformazione } \\
\text { totale }\end{array}$}} & \multirow{3}{*}{$\begin{array}{l}\text { Incremento } \\
\text { della defor. } \\
\text { riferito } \\
\text { ad } 1 \text { igr. di } \\
\text { incremento } \\
\text { dello sforzo }\end{array}$} \\
\hline & & & \multirow{2}{*}{ 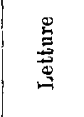 } & \multirow{2}{*}{ 总: } & \multirow{2}{*}{ 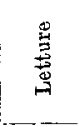 } & \multirow{2}{*}{ 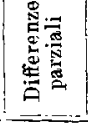 } & & & & \\
\hline & & & & & & & & 4) & ) & \\
\hline & & & & .. & & .. & & & & $\cdots$ \\
\hline febbraio & 9 & 0 & 834 & - & 1893 & - & - & 0 & & - \\
\hline$\gg$ & & 100 & 939 & +105 & 2021 & +128 & +233 & 233 & & +2.33 \\
\hline$\gg$ & & 200 & 1060 & +121 & 2138 & +117 & +238 & 471 & & $+\mathbf{2 . 3 8}$ \\
\hline$»$ & & 400 & 1299 & +239 & 2375 & +237 & +476 & 947 & & +238 \\
\hline " & & 600 & 1638 & +239 & 2618 & +243 & +482 & 1429 & & +2.41 \\
\hline 》 & & 800 & 1779 & +241 & 2867 & +249 & +490 & 1919 & & +2.45 \\
\hline " & & 1000 & 2025 & +246 & 3119 & +252 & +498 & 2417 & & +2.49 \\
\hline$\gg$ & & 1200 & 2272 & +247 & 3371 & +252 & +499 & 2916 & & +2.50 \\
\hline$"$ & & 1100 & 2154 & -118 & 3255 & -116 & -234 & 2682 & & -2.34 \\
\hline$»$ & & 900 & 1917 & -237 & 3015 & -240 & -477 & 2205 & & -2.38 \\
\hline » & & 700 & 1682 & -235 & 2772 & -243 & -478 & 1727 & & -2.39 \\
\hline
\end{tabular}

1) Contata upartire allinizio dellesperienza - Riferita allo stato speciale in cui il materiale viene a trovarsi alla fine delle operazioni preparatorie descritte al principio di questa nota. 


\begin{tabular}{|c|c|c|c|c|c|c|c|c|c|c|}
\hline \multirow{3}{*}{ Giorno } & \multirow{3}{*}{ Ora } & \multirow{3}{*}{ 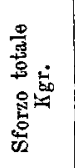 } & \multicolumn{4}{|c|}{ Specchio sinistro, Specchio destro } & \multirow{3}{*}{ 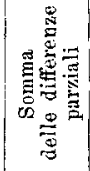 } & \multirow{2}{*}{\multicolumn{2}{|c|}{$\begin{array}{c}\text { Deformazione } \\
\text { totale }\end{array}$}} & \multirow{3}{*}{$\begin{array}{l}\text { Incremento } \\
\text { della defor. } \\
\text { riferito } \\
\text { ad l kgr. di } \\
\text { incremento } \\
\text { dello sforzo }\end{array}$} \\
\hline & & & \multirow{2}{*}{ 总 } & \multirow{2}{*}{ 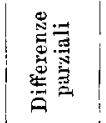 } & \multirow{2}{*}{ 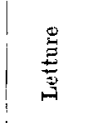 } & \multirow{2}{*}{ 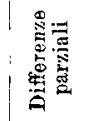 } & & & & \\
\hline & & & & & & & & 1) & 2) & \\
\hline & & & & & & & & & & \\
\hline 2 febbraio & & 500 & 1444 & -238 & 2529 & -243 & -481 & 1246 & & -2.41 \\
\hline$\Rightarrow$ & & 300 & 1204 & -240 & 2282 & -247 & -487 & 759 & & -2.43 \\
\hline - & & 100 & 955 & -249 & 2033 & -249 & -498 & 261 & & -2.49 \\
\hline » & & 200 & 1073 & +118 & 2150 & +117 & +235 & 496 & & $+\mathbf{2 . 3 5}$ \\
\hline » & & 400 & 1313 & +240 & 2386 & +236 & +476 & 972 & & $+2.3 x$ \\
\hline 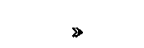 & & 600 & $1551^{\prime}$ & +238 & 2625 & +239 & +477 & 1449 & & $\begin{array}{r}+2.39 \\
\end{array}$ \\
\hline 8 & & 800 & 1790 & +239 & 2870 & +245 & +484 & 1933 & & +2.42 \\
\hline i & & 1000 & 2029 & +239 & $\$ 119$ & +249 & +488 & 2421 & & $\begin{array}{r}+2.44 \\
\end{array}$ \\
\hline 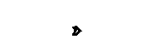 & & 900 & 1911 & -118 & 3001 & -118 & -236 & 2185 & & -2.36 \\
\hline : & & 700 & $1679:$ & -232 & 2761 & -240 & -472 & 1713 & & -2.36 \\
\hline$\triangle$ & & 500 & 1441 & -238 & 2520 & -241 & -479 & 1254 & & -2.39 \\
\hline * & & 300 & $1200^{\prime}$ & -241 & 2274 & -246 & -487 & 747 & & -2.44 \\
\hline » & & 400 & 1318 , & +118 & 2390 & +116 & +234 & 981 & & $+\mathbf{2 . 3 4}$ \\
\hline » & & 600 & 1554 & $+23 i$ & 2630 & +240 & +476 & 1457 & & +2.38 \\
\hline 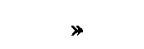 & & 800 & 1790 & +236 & 2872 & +242 & +478 & 1035 & & +2.39 \\
\hline 》 & & 700 & 1674 & -116 & 2750 & -117 & -233 & 1702 & & $-\mathbf{2 . 3 3}$ \\
\hline$\triangleright$ & & 500 & 1439 & -230 & 2516 & -239 & -474 & 1228 & & $\therefore 2.37$ \\
\hline 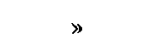 & & $\overline{600}$ & 1557 & -118 & 2632 & +116 & +234 & 1462 & 0 & $+\mathbf{2 . 8 3}$ \\
\hline $\overrightarrow{\rangle}$ & & 700 & 1673. & +116 & $2 \overline{152}$ & +120 & +236 & 1698 & 236 & +2.36 \\
\hline 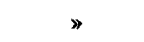 & & 800 & 1791 & +118 & 2873 & +-121 & +239 & 1937 & 475 & +239 \\
\hline 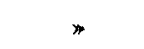 & & 1000 & 2029 & +238 & 3118 & +-245 & +483 & 2420 & 958 & +2.41 \\
\hline * & & 1200 & 2275 & +246 & 3367 & +249 & +495 & 2915 & 1453 & +2.48 \\
\hline$*$ & & 1100 & 2157 & -118 & 3250 & -117 & -235 & 2680 & 1218 & -2.35 \\
\hline * & & 1000 & 2040 & -117 & 3132 & -118 & -235 & 2445 & 983 & -2.35 \\
\hline 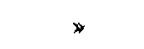 & & 800 & 1801 & -239 & 2891 & -241 & -480 & 1965 & 503 & -2.40 \\
\hline$\geqslant$ & & 600 & 1564 & -237 & 2646 & -245 & -482 & 1483 & 21 & -2.41 \\
\hline * & & 700 & 1681 & +117 & 2764 & +-118 & +285 & 1718 & 256 & +2.35 \\
\hline 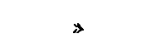 & & 800 & 1798 & +117 & 2885 & $|+121|$ & +238 & 1956 & 494 & +2.38 \\
\hline$m$ & & 1000 & $2038 !$ & +240 & 3125 & +240 & +480 & 2436 & 974 & +2.40 \\
\hline * & & 1200 & 2277 & +239 & 3367 & +242 & +481 & 2917 & 1455 & +2.41 \\
\hline 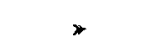 & & 1100 & 2159 & $\mid-118$ & 3251 & -116 & -234 & 2683 & 1221 & -2.34 \\
\hline * & & 1000 & 2040 & -119 & 3134 & -117 & -236 & 2447 & 985 & -2.36 \\
\hline$\Rightarrow$ & & 800 & 1804 & -236 & 2890 & -244 & -480 & 1967 & 505 & -2.40 \\
\hline × & & 600 & 1566 & -238 & 2646 & -244 & -482 & 1485 & 23 & -2.41 \\
\hline$\triangleright$ & & 400 & 1328 & -238 & 2401 & -245 & -483 & 1002 & -460 & $0-2.42$ \\
\hline » & & 200 & 1085 & -243 & 2155 & $|-246|$ & -489 & 513 & -948 & $9:-2.44$ \\
\hline
\end{tabular}




\begin{tabular}{|c|c|c|c|c|c|c|c|c|c|c|}
\hline \multirow[b]{2}{*}{ Giorno } & \multirow[b]{2}{*}{ Ora } & \multirow{2}{*}{ 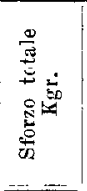 } & \multicolumn{4}{|c|}{ Specchio sinistro Specclio destro } & \multirow[b]{2}{*}{ 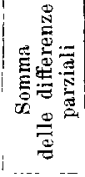 } & \multirow{2}{*}{\multicolumn{2}{|c|}{$\begin{array}{c}\text { Deformazione } \\
\text { totale }\end{array}$}} & \multirow{2}{*}{$\begin{array}{l}\text { Increment } \\
\text { della defor } \\
\text { riferito } \\
\text { ad l kgr. } \\
\text { increment } \\
\text { dello sforz }\end{array}$} \\
\hline & & & 兽 & 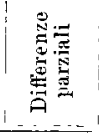 & 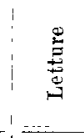 & 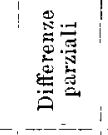 & & & & \\
\hline 2 febbraio & & 0 & 838 & -247 & 1909 & -246 & -493 & & $0-1442$ & -2.47 \\
\hline 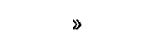 & 14 & 0 & $830 \overline{0}$ & -8 & 1903 & -6 & -14 & & $6-1456$ & - \\
\hline . & & $\cdots$ & $\cdots$ & $\cdots$ & . & $\cdots$ & $\cdots$ & $\cdots$ & .. & \\
\hline 10 febbraio & 15 & 600 & 1609 & - & 2640 & - & - & & 0 & - \\
\hline$\gg$ & & 700 & 1725 & +116 & 2758 & +118 & +234 & & $\mathbf{2 3 4}$ & +2.34 \\
\hline$»$ & & 600 & 1609 & -116 & 2641 & -117 & $|-233|$ & & 1 & -2.33 \\
\hline » & & 500 & 1493 & -116 & 2521 & -120 & -236 & & - 285 & -2.36 \\
\hline$\gg$ & & 600 & 1609 & +116 & 2639 & +118 & +234 & & - & $1+2.34$ \\
\hline » & & 700 & 1725 & +116 & 2758 & +119 & +235 & & 234 & +2.35 \\
\hline » & & 800 & 1841 & +116 & 2877 & +119 & +235 & & 469 & $+2.3 \tilde{5}$ \\
\hline 》 & & 900 & 1959 & +118 & 2997 & +120 & +238 & & 707 & +2.38 \\
\hline » & & 800 & 1843 & -116 & 2880 & -117 & -233 & & 474 & -2.33 \\
\hline » & & 600 & 1609 & -234 & 2644 & $\mid-236$ & -470 & & 4 & -2.35 \\
\hline$»$ & & 400 & $1376^{\circ}$ & -233 & 2401 & -240 & -473 & & -496 & -2.37 \\
\hline » & & 300 & 1257 & -119 & 2284 & $\mid-120$ & -239 & & -708 & -2.39 \\
\hline 》 & & $400^{\prime}$ & 1375 & +118 & 2399 & +115 & +233 & & -475 & +2.33 \\
\hline » & & 600 & 1607 & +232 & 2636 & +237 & +469 & & - & +2.34 \\
\hline 》 & & 800 & 1840 & +233 & 2875 & +239 & +472 & & 466 & +2.36 \\
\hline » & & 900 & 1958 & +118 & 2997 & +122 & +240 & & 706 & +2.40 \\
\hline$"$ & & 1000 & 2077 & +119 & 3118 & $|+121|$ & +240 & & 946 & +2.40 \\
\hline " & & 1200 & 2319 & +242 & 3367 & $\mid+249$ & +491 & & 1437 & +2.46 \\
\hline " & & 1100 & 2203 & -116 & 3249 & -118 & -234 & & 1203 & -2.34 \\
\hline$\gg$ & & 1000 & 2086 & -117 & 3130 & -119 & -236 & & 967 & -2.36 \\
\hline$»$ & & 800 & 1850 & -236 & 2892 & -238 & -474 & & 493 & -2.37 \\
\hline 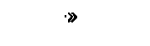 & & 600 & 1616 & -234 & 2650 & -242 & -476 & & 17 & -2.38 \\
\hline * & & 400 & 1380 & -236 & 2408 & -242 & -478 & & -461 & -2.39 \\
\hline$\gg$ & & 200 & 1143 & -237 & 2165 & -243 & -480 & & -941 & -2.40 \\
\hline 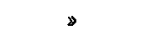 & & 0 & 899 & -244 & 1912 & -253 & -497 & & $-14 \mathrm{~g} 8$ & -2.48 \\
\hline » & & 100 & 1016 & +117 & 2030 & +118 & +285 & & -1203 & $+\mathbf{2 . 3 5}$ \\
\hline » & & 200 & 1135 & +119 & 2147 & +117 & +236 & & -967 & +2.36 \\
\hline$\$$ & & 400 & 1371 & +236 & 2383 & +236 & +472 & & -495 & +2.36 \\
\hline$"$ & & $600^{\prime}$ & 1606 & +235 & 2622 & $|+239|$ & +474 & & $-\quad 21$ & +2.37 \\
\hline " & & 800 & 1843 & +237 & 2865 & +243 & +480 & & 459 & +2.40 \\
\hline 》 & & 1000 & 2079 & +236 & 3110 & +245 & +481 & & 940 & +2.41 \\
\hline$"$ & & 1200 & 2324 & +245 & 3360 & +250 & +495 & & 1485 & +2.47 \\
\hline$\therefore$ & & . & $\cdots$ & .. & & & & & & \\
\hline
\end{tabular}

